\title{
POLYZÊTAS STRICTS, LARGES ET PONDÉRÉS
}

\author{
par
}

Jacky Cresson

\begin{abstract}
Résumé. - Nous explicitons des formules de passage entre les polyzêtas stricts et larges. On utilise le formalisme des séries formelles non-commutatives. Ces formules reposent sur la définition de la substitution de deux séries formelles non-commutatives.
\end{abstract}

Abstract. - We derive explicit formulae connecting large and strict multiple zeta values. We use the formalism of non-commutative formal power series and a non-commutative analogue of the usual substitution for formal power series.

\section{Table des matières}

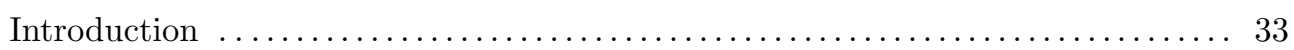

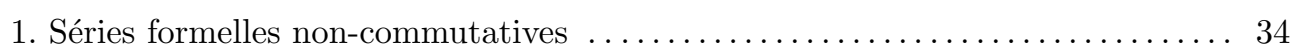

2. Autour d'un résultat d'Ulanskiŭ $\ldots \ldots \ldots \ldots \ldots \ldots \ldots \ldots \ldots \ldots \ldots \ldots \ldots \ldots$

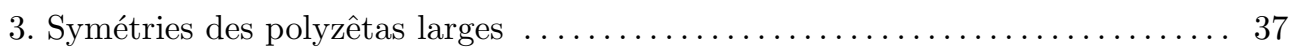

4. Régularisation des polyzêtas larges $\ldots \ldots \ldots \ldots \ldots \ldots \ldots \ldots \ldots \ldots \ldots \ldots \ldots \ldots$

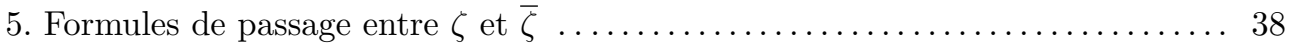

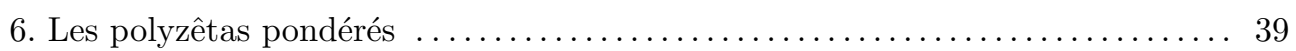

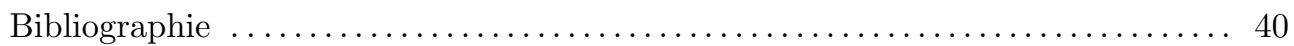

\section{Introduction}

Une généralisation de la fonction zêta de Riemann $\zeta(s)$ est donnée par les séries polyzêtas, définies pour tout entier $p \geq 1$ et tout $p$-uplet $\mathbf{s}=\left(s_{1}, s_{2}, \ldots, s_{p}\right)$ d'entiers $\geq 1$, avec $s_{1} \geq 2$, par

$$
\zeta\left(s_{1}, s_{2}, \ldots, s_{p}\right)=\sum_{k_{1}>k_{2}>\cdots>k_{p} \geq 1} \frac{1}{k_{1}^{s_{1}} k_{2}^{s_{2}} \cdots k_{p}^{s_{p}}} .
$$

Les entiers $p$ et $s_{1}+s_{2}+\cdots+s_{p}$ sont respectivement la profondeur et le poids de $\zeta\left(s_{1}, s_{2}, \ldots, s_{p}\right)$.

La nature arithmétique de ces séries est aussi peu connue que celle des nombres $\zeta(s)$. On renvoie à [11] pour un survol du sujet.

On connait néanmoins un certain nombre de résultats diophantiens sur les nombres zêtas de Riemann, i.e en profondeur 1 :

(i) Le nombre $\zeta(3)$ est irrationnel (Apéry [1]); 
(ii) La dimension de l'espace vectoriel engendré sur $\mathbb{Q}$ par $1, \zeta(3), \zeta(5), \ldots, \zeta(A)$ (avec $A$ impair) croît au moins comme $\log (A)([\mathbf{3}, \mathbf{1 4}])$;

(iii) Au moins un des quatre nombres $\zeta(5), \zeta(7), \zeta(9), \zeta(11)$ est irrationnel (Zudilin [19]).

Ces résultats peuvent être obtenus par l'étude de certaines séries de la forme

$$
\sum_{k=1}^{\infty} \frac{P(k)}{k^{A}(k+1)^{A} \cdots(k+n)^{A}} z^{-k}
$$

avec $P(X) \in \mathbb{Q}[X], n \geq 0, A \geq 1$ et $|z| \geq 1$.

Les divers choix de $P$ conduisent à des séries hypergéométriques généralisées (voir [2, 15]).

Dans $([\mathbf{7}, \mathbf{8}])$ nous proposons une généralisation de cette méthode hypergéométrique en profondeur quelconque en considérant a priori des séries multiples de la forme

$$
\sum_{k_{1} \geq \cdots \geq k_{p} \geq 1} \frac{P\left(k_{1}, \ldots, k_{p}\right)}{\left(k_{1}\right)_{n_{1}+1}^{A_{1}} \cdots\left(k_{p}\right)_{n_{p}+1}^{A_{p}}} z_{1}^{-k_{1}} \cdots z_{p}^{-k_{p}},
$$

avec $P\left(X_{1}, \ldots, X_{p}\right) \in \mathbb{Q}\left[X_{1}, \ldots, X_{p}\right]$, des entiers $A_{j} \geq 2$ et $n_{j} \geq 0$ et $\left|z_{1}\right| \geq 1, \ldots,\left|z_{p}\right| \geq 1$, et où $(\alpha)_{m}=\alpha(\alpha+1) \cdots(\alpha+m-1)$ désigne le symbole de Pochhammer.

Lorsque $z_{1}=\cdots=z_{p}=1$ ces séries sont des combinaisons linéaires à coefficients dans $\mathbb{Q}$ de polyzêtas larges définis par

$$
\bar{\zeta}\left(s_{1}, s_{2}, \ldots, s_{p}\right)=\sum_{k_{1} \geq k_{2} \geq \cdots \geq k_{p} \geq 1} \frac{1}{k_{1}^{s_{1}} k_{2}^{s_{2}} \cdots k_{p}^{s_{p}}} .
$$

On renvoie à $[\mathbf{7}]$ pour plus de détails.

Via des calculs élémentaires on peut décomposer tout polyzêtas larges $\bar{\zeta}$ suivant les polyzêtas stricts $\zeta$ (voir par exemple [16]). La formule de décomposition est par ailleurs explicite (voir le §.2). Les polyzêtas stricts possèdent de nombreuses propriétés algébriques (voir [17]). On peut se demander comment ces propriétés sont transformées par passage aux polyzêtas larges.

Pour aborder cette question, nous explicitons la formule de passage entre les polyzetas stricts et larges via les séries génératrices associées. Le cadre de travail est celui des séries formelles non-commutatives. On introduit à cette occasion l'opération de substitution de deux séries formelles non-commutatives. Ce formalisme nous permet de mieux mettre en évidence la manière dont les symétries satisfaites par les polyzetas stricts sont modifiées. L'étude de ces symétries nous conduit à introduire une modification naturelle des polyzetas stricts, les polyzêtas pondérés, dont les symétries sont d'une complexité minimale.

\section{Séries formelles non-commutatives}

Soit $Y=\left\{y_{i}\right\}_{i \in \mathbb{N}}$ un alphabet. On note $Y^{*}$ l'ensemble des mots construis sur $Y$, comprenant le mot vide noté $\emptyset$. Un élément de $Y^{*}$ est noté $y_{\mathbf{s}}=y_{s_{1}} \ldots y_{s_{p}}, p \geq 1, s_{i} \in \mathbb{N}^{*}$. L'entier $p$ est la longueur de $y_{\mathbf{s}}$, notée aussi $l(\mathbf{s})$. La concaténation de deux mots $y_{\mathbf{s}}$ et $y_{\mathbf{u}}$ de $Y^{*}$ avec $\mathbf{s}=\left(s_{1}, \ldots, s_{p}\right)$, $\mathbf{u}=\left(u_{1}, \ldots, u_{q}\right)$, est le mot noté $y_{\mathbf{s}} y_{\mathbf{u}}$ défini par $y_{\mathbf{s u}}$ où $\mathbf{s u}=\left(s_{1}, \ldots, s_{p}, u_{1}, \ldots, u_{q}\right)$.

Soit $\mathrm{K}$ un anneau, on note $\mathrm{K}\langle Y\rangle$ l'ensemble des séries formelles non-commutatives à coefficients dans $\mathrm{K}$ construites sur $Y$.

Étant donnée une application $\mathrm{L}: Y^{*} \rightarrow \mathrm{K}$, on note $\mathrm{L}_{\mathbf{s}}$ l'évaluation de $\mathrm{L}$ sur le mot $y_{\mathbf{s}} \in Y^{*}$. Ce type d'applications est un exemple de moule dans la terminologie de Jean Ecalle [6]. 
À toute application $\mathrm{L}: Y^{*} \rightarrow \mathrm{K}$, on associe l'élément de $\mathrm{K}\langle Y\rangle$, noté $\Phi_{\mathrm{L}}$ et défini par

$$
\Phi_{\mathrm{L}}=\sum_{y_{\mathbf{s}} \in Y^{*}} \mathrm{~L}_{\mathbf{s}} y_{\mathbf{s}}
$$

appelé série génératrice de $\mathrm{L}$.

On associe à toute lettre $y_{s} \in Y$ un poids, noté $\left|y_{s}\right|$ et défini par $\left|y_{s}\right|=s$. Le poids d'un mot $y_{\mathbf{s}} \in Y^{*}$ est défini par $\left|y_{\mathbf{s}}\right|=s_{1}+\cdots+s_{p}$.

L'ensemble $\mathrm{K}\langle Y\rangle$ est gradué par le poids. Cette graduation respecte l'opération de concaténation des mots. Une composante homogène de degré $r \in \mathbb{N}^{*}$ d'une série non-commutative $\Phi=\sum_{y_{\mathbf{s}} \in Y^{*}} \mathrm{M}_{\mathbf{s}} y_{\mathbf{s}}$, $\mathrm{M}_{\mathbf{s}} \in K$ notée $\Phi_{r}$ est donnée par

$$
\Phi_{r}=\sum_{y_{\mathbf{s}} \in Y^{*},\left|y_{\mathbf{s}}\right|=r} \mathrm{M}_{\mathbf{s}} y_{\mathbf{s}}
$$

L'ensemble $\mathrm{K}\langle Y\rangle$ possède une structure d'algèbre, analogue non-commutatif de la structure d'algèbre sur les séries formelles commutatives.

La graduation permet de définir un analogue non-commutatif de la substitution des séries formelles de la manière suivante :

Définition 1. - Soient $\Phi_{\mathrm{M}}$ et $\Phi_{\mathrm{N}}$ deux séries de $\mathrm{K}\langle Y\rangle$. On note $\Phi_{\mathrm{M}} \circ \Phi_{\mathrm{N}}$ la série génératrice définie par

$$
\Phi_{\mathrm{M}} \circ \Phi_{\mathrm{N}}=\sum_{r \geq 0} \sum_{\mathbf{s}=\left(s_{1}, \ldots, s_{r}\right),} \mathrm{M}_{s_{i} \in \mathbb{N}^{*}}\left(\Phi_{\mathrm{N}}\right)_{s_{1}} \ldots\left(\Phi_{\mathrm{N}}\right)_{s_{p}},
$$

dans $\mathrm{K}\langle Y\rangle$, où $\left(\Phi_{\mathrm{N}}\right)_{s}, s \in \mathbb{N}$ est la composante homogène de poids $s$ de $\Phi_{\mathrm{N}}$.

Il est possible de calculer explicitement les coefficients de la série $\Phi_{\mathrm{M}} \circ \Phi_{\mathrm{N}}$ notés $(\mathrm{M} \circ \mathrm{N})_{\mathbf{s}}$.

Lemme 1. - Pour tout $\mathbf{s}=\left(s_{1}, \ldots, s_{r}\right), s_{i} \in \mathbb{N}^{*}$ on a

$$
(\mathrm{M} \circ \mathrm{N})_{\mathbf{s}}=\sum_{\mathbf{s}^{1} \ldots \mathbf{s}^{k}=\mathbf{s}} \mathrm{M}_{\left(\left|\mathbf{s}^{1}\right|, \ldots,\left|\mathbf{s}^{k}\right|\right)} \mathrm{N}_{\mathbf{s}^{1}} \ldots \mathrm{N}_{\mathbf{s}^{k}}
$$

où $k \geq 1$ et $\mathbf{s}^{i} \neq \emptyset$ et $|\mathbf{s}|=s_{1}+\cdots+s_{p}$ pour tout $\mathbf{s}=s_{1} \ldots s_{p}$.

Démonstration. - L'équation (4) est équivalente à

$$
\text { (6) } \Phi_{\mathrm{M}} \circ \Phi_{\mathrm{N}}=\sum_{r \geq 0} \sum_{\mathbf{u}=\left(u_{1}, \ldots, u_{r}\right), u_{i} \in \mathbb{N}^{*}} \mathrm{M}_{\mathbf{u}}\left(\sum_{y_{\mathbf{s}} \in Y^{*},\left|y_{\mathbf{s}}\right|=u_{1}} \mathrm{~N}_{\mathbf{s}} y_{\mathbf{s}}\right) \ldots\left(\sum_{y_{\mathbf{s}} \in Y^{*},\left|y_{\mathbf{s}}\right|=u_{r}} \mathrm{~N}_{\mathbf{s}} y_{\mathbf{s}}\right) \text {. }
$$

Un mot $y_{\mathbf{s}}$ étant fixé, toute partition de $y_{\mathbf{s}}$ de la forme $y_{\mathbf{s}}=y_{\mathbf{s}^{1}} \ldots y_{\mathbf{s}^{k}}, k=1, \ldots, l(\mathbf{s})$, intervient dans la somme (6) avec un coefficient de la forme

$$
\mathrm{M}_{\left(u_{1}, \ldots, u_{k}\right)} \mathrm{N}_{\mathbf{s}^{1}} \ldots \mathrm{N}_{\mathbf{s}^{k}}
$$

où $u_{i}=\left|\mathbf{s}^{i}\right|$. En regroupant ces termes, on obtient la formule (5) pour le coefficients de $\Phi_{\mathrm{M}} \circ \Phi_{\mathrm{N}}$ en $y_{\mathbf{s}}$.

Remarque 1. - L'opération o n'est pas usuelle dans l'étude combinatoire des séries formelles non-commutatives (voir [13] pour une présentation des techniques habituelles). La formule (5) intervient dans le formalisme des moules développé par Jean Écalle comme loi de composition sur les moules (voir [6]).

Lemme 2. - L'élément neutre pour la loi $\circ$ est la série $\mathrm{I}=\sum_{s \in \mathbb{N}} y_{s}$. 
La démonstration est immédiate en utilisant (5).

On peut caractériser les séries inversibles pour o, ce qui nous sera utile dans la suite.

Lemme 3. - Les éléments inversibles de $\mathrm{K}\langle Y\rangle$ pour la loi $\circ$ sont les séries $\Phi_{\mathrm{M}}=\sum_{y_{\mathbf{s}} \in Y^{*}} \mathrm{M}_{\mathbf{s}} y_{\mathbf{s}}$ telles que $\mathrm{M}_{\emptyset}=0, \mathrm{M}_{s} \neq 0$ pour tout $s \in \mathbb{N}^{*}$.

Démonstration. - Soit $\Phi_{\mathrm{M}}=\sum_{y_{\mathbf{s}} \in Y^{*}} \mathrm{M}_{\mathrm{s}} y_{\mathrm{s}}$ un élément inversible de $\mathrm{K}\langle Y\rangle$. Il existe donc $\Phi_{\mathrm{N}}=$ $\sum_{y_{\mathrm{s}} \in Y^{*}} \mathrm{~N}_{\mathrm{s}} y_{\mathrm{s}}$ telle que $\mathrm{I}=\Phi_{\mathrm{M}} \circ \Phi_{\mathrm{N}}$. Les coefficients de la série $\Phi_{N}$ se déterminent par récurrence sur la longueur des suites $\mathbf{s}$. En effet, en appliquant la formule (5), on a pour toute suite $\mathbf{s}$ :

$$
\mathrm{M}_{|\mathbf{s}|} \mathrm{N}_{\mathbf{s}}=\mathrm{I}^{\mathrm{s}}-\sum_{\mathbf{s}^{1} \ldots \mathbf{s}^{k}=\mathbf{s}, k>1} \mathrm{M}_{\left(\left|\mathbf{s}^{1}\right|, \ldots,\left|\mathbf{s}^{k}\right|\right)} \mathrm{N}_{\mathbf{s}^{1}} \ldots \mathrm{N}_{\mathbf{s}^{k}} .
$$

Le membre de droite contient des coefficients $\mathrm{N}_{\mathbf{s}^{\prime}}$ sur des suites $\mathbf{s}^{\prime}$ de longueur strictement inférieure à $l(\mathbf{s})$. Cette équation détermine donc $\mathrm{N}_{\mathbf{s}}$ si et seulement si $\mathrm{M}_{|\mathbf{s}|} \neq 0$. Comme $|\mathbf{s}| \in \mathbb{N}^{*}$, on en déduit le lemme.

\section{Autour d'un résultat d'Ulanskiı̌}

Dans ([16, p. 577, Proposition 2]) Ulanskiǔ donne une formule de décomposition qui peut se formuler comme suit :

Lemme 4. - Pour toute suite $\mathbf{s}=\left(s_{1}, \ldots, s_{r}\right), s_{i} \in \mathbb{N}^{*}, s_{1} \geq 2$, on a

$$
\bar{\zeta}_{\mathbf{s}}=\sum_{\mathbf{s}^{1}, \ldots \mathbf{s}^{k}=\mathbf{s}} \zeta_{\left|\mathbf{s}^{1}\right|, \ldots,\left|\mathbf{s}^{k}\right|}
$$

où $k \geq 1, \mathbf{s}^{i} \neq \emptyset$.

Démonstration. - La démonstration repose sur l'étude des polyzêtas mixtes de la forme

$$
\stackrel{\otimes}{\zeta}_{\mathbf{s}}(i)=\sum_{n_{1}>\cdots>n_{i} \geq n_{i+1} \geq \ldots n_{p} \geq 1} \frac{1}{n_{1}^{s_{1}} \ldots n_{p}^{s_{p}}},
$$

avec $i=1, \ldots, l(\mathbf{s})$.

On a $\stackrel{\otimes}{\zeta}_{\mathbf{s}}(1)=\bar{\zeta}_{\mathbf{s}}$ et $\stackrel{\otimes}{\zeta}_{\mathbf{s}}(l(\mathbf{s}))=\zeta_{\mathbf{s}}$. Un simple calcul donne

$$
\stackrel{\otimes}{\zeta}_{s_{1} \ldots s_{p}}(i)=\stackrel{\otimes}{\zeta}_{s_{1} \ldots s_{p}}(i+1)+\stackrel{\otimes}{\zeta}_{s_{1}, \ldots, s_{i-1}, s_{i}+s_{i+1}, s_{i+2}, \ldots, s_{p}}(i),
$$

pour $i<p$.

On démontre par récurrence sur la longueur des suites la formule

$$
\stackrel{\otimes}{\zeta}_{\mathbf{s}=s_{1} \ldots s_{r}}(i)=\sum_{\mathbf{s}^{1} \ldots \mathbf{s}^{k}=s_{i}, \ldots, s_{r}} \zeta_{s_{1}, \ldots, s_{i-1},\left|\mathbf{s}^{1}\right|, \ldots,\left|\mathbf{s}^{k}\right|}
$$

On remarque que la formule (4) ressemble beaucoup à une opération de composition. Mais pour écrire correctement cette composition il est nécessaire de définir $\bar{\zeta}$ pour tous les s, i.e. sur les divergents où $s_{1}=1$. Pour ce faire, nous allons étudier les symétries des polyzêtas larges et expliciter une formule de régularisation combinatoire. 


\section{Symétries des polyzêtas larges}

On note $Y_{c}$ le sous-ensemble de $Y^{*}$ constitué des mots $y_{\mathbf{s}}=y_{s_{1}} \ldots y_{s_{r}}$ tels que $s_{1} \geq 2$, c'est à dire tels que $\zeta_{\mathbf{s}}$ et $\bar{\zeta}_{\mathbf{s}}$ soient convergents.

L'écriture sous la forme de série des polyzêtas stricts permet de mettre en évidence des symétries, appelées symétries de battage contractant (voir [17]). On a par exemple

$$
\zeta_{s} \zeta_{u}=\zeta_{s, u}+\zeta_{u, s}+\zeta_{s+u}
$$

La généralisation de cette formule pour des suites quelconques fait intervenir le produit stuffle, noté $\star$ sur les lettres, défini par récurrence via les relations $y_{\mathbf{s}} \star \emptyset=y_{\mathbf{s}}, \emptyset \star y_{\mathbf{s}}=y_{\mathbf{s}}$ et

$$
y_{s} y_{\mathbf{s}} \star y_{u} y_{\mathbf{u}}=y_{s}\left(y_{\mathbf{s}} \star y_{u} y_{\mathbf{u}}\right)+y_{u}\left(y_{s} y_{\mathbf{s}} \star y_{\mathbf{u}}\right)+y_{s+u}\left(y_{\mathbf{s}} \star y_{\mathbf{u}}\right) \text {. }
$$

On a pour tout $y_{\mathbf{s}} \in Y_{c}$ et $y_{\mathbf{u}} \in Y_{c}$

$$
\zeta\left(y_{\mathbf{s}}\right) \zeta\left(y_{\mathbf{u}}\right)=\zeta\left(y_{\mathbf{s}} \star y_{\mathbf{u}}\right) .
$$

Les polyzêtas larges vérifient aussi une relation de symétrie, beaucoup moins jolie, et qui s'obtient par transport de la loi $\star$ par l'isomorphisme linéaire $\phi$ défini par $\phi\left(y_{s}\right)=y_{s}$ pour tout $s \in \mathbb{N}$ et

$$
\phi\left(y_{s_{1}} \ldots y_{s_{p}}\right)=y_{s_{1}} \phi\left(y_{s_{2}} \ldots y_{s_{p}}\right)+\phi\left(y_{s_{1}+s_{2}} y_{s_{3}} \ldots y_{s_{p}}\right)
$$

pour tout $p \geq 2$.

Cette relation traduit la relation de récurrence (8) sur les polyzêtas mixtes. On a donc pour tout $\operatorname{mot} y_{\mathbf{s}}$ de $Y_{c}$ :

$$
\bar{\zeta}\left(y_{\mathbf{s}}\right)=\zeta\left(\phi\left(y_{\mathbf{s}}\right)\right)
$$

On note $\star_{\phi}$ la loi tordue sur $\mathbb{R}\langle Y\rangle$ définie par

$$
\phi\left(y_{\mathbf{s}} \star_{\phi} y_{\mathbf{u}}\right)=\phi\left(y_{\mathbf{s}}\right) \star \phi\left(y_{\mathbf{u}}\right) .
$$

Le symétrie stuffle (12) pour les polyzêtas stricts et la relation de structure (13) impliquent :

Lemme 5. - Pour tous mots $y_{\mathbf{s}}, y_{\mathbf{u}}$ de $Y_{c}$ on a $\bar{\zeta}\left(y_{\mathbf{s}}\right) \bar{\zeta}\left(y_{\mathbf{u}}\right)=\bar{\zeta}\left(y_{\mathbf{s}} \star_{\phi} y_{\mathbf{u}}\right)$.

Démonstration. - On a $\bar{\zeta}\left(y_{\mathbf{s}}\right) \bar{\zeta}\left(y_{\mathbf{u}}\right)=\zeta\left(\phi\left(y_{\mathbf{s}}\right)\right) \zeta\left(\phi\left(y_{\mathbf{u}}\right)\right)$. Comme $\zeta$ vérifie la symétrie $\star$, on obtient $\bar{\zeta}\left(y_{\mathbf{s}}\right) \bar{\zeta}\left(y_{\mathbf{u}}\right)=\zeta\left(\phi\left(y_{\mathbf{s}}\right) \star \phi\left(y_{\mathbf{u}}\right)\right)$. Le lemme découle alors de $(14)$.

La symétrie $\star_{\phi}$ est beaucoup complexe que la symétrie $\star$. En suivant la démarche ci-dessus, on peut introduire une famille à un paramètre d'isomorphismes linéaires $\phi_{\lambda}, \lambda \in \mathbb{R}$ et déformer continuement la symétrie des polyzêtas stricts, pour passer par exemple d'une symétrie stuffle à une symétrie shuffle (voir §.6). On obtient alors les algèbres de Hoffman tordues [18].

\section{Régularisation des polyzêtas larges}

Nous avons pour le moment travaillé sur le sous ensemble des mots convergents $Y_{c} \subset Y^{*}$. L'existence d'une symétrie $\star_{\phi}$ permet, comme dans le cas strict (voir [12]), de donner un sens aux polyzêtas larges divergents.

Lemme 6. - Soit $\gamma \in \mathbb{R}$. Il existe une unique extension $\bar{\zeta}_{\star_{\phi}}$ de $\bar{\zeta}$ sur $Y^{*}$ telle que $\bar{\zeta}_{\star_{\phi}}\left(y_{1}\right)=\gamma$ et $\bar{\zeta}_{\star_{\phi}}\left(y_{\mathbf{s}} \star_{\phi} y_{\mathbf{u}}\right)=\bar{\zeta}_{\star_{\phi}}\left(y_{\mathbf{s}}\right) \bar{\zeta}_{\star_{\phi}}\left(y_{\mathbf{u}}\right)$.

Démonstration. - La démonstration repose sur le calcul de $y_{1} \star_{\phi} y_{1}^{n} y_{\mathbf{s}}$, pour $n \geq 0, y_{\mathbf{s}} \in Y_{c}$. Pour tout $n \geq 0$, on a

$$
y_{1} \star_{\phi} y_{1}^{n} y_{\mathbf{s}}=\phi^{-1}\left(\phi\left(y_{1}\right) \star \phi\left(y_{1}^{n} y_{\mathbf{s}}\right)\right)
$$


Comme $\phi\left(y_{1}\right)=y_{1}$, et $\phi^{-1}\left(y_{\mathbf{u}}\right)=y_{\mathbf{u}}+\ldots$, où $\ldots$ représente des mots de longueur $<l\left(y_{\mathbf{u}}\right)$, on en déduit que l'équation (15) s'écrit

$$
y_{1} \star_{\phi} y_{1}^{n} y_{\mathbf{s}}=y_{1}^{n+1} y_{\mathbf{s}}+\ldots,
$$

où ... représente des mots de $Y_{c}$ ou de la forme $1^{i} y_{\mathbf{v}}$ avec $i \leq n$ et $y_{\mathbf{v}} \in Y_{c}$.

Soit $\bar{\zeta}_{\star_{\phi}}\left(y_{1}\right)=\gamma, \gamma \in \mathbb{R}$ une constante. En utilisant l'équation (16), il est possible de déterminer par récurrence la valeur de $\bar{\zeta}_{\star_{\phi}}\left(y_{1}^{n} y_{\mathbf{s}}\right)$ pour tout $y_{\mathbf{s}} \in Y_{c}$. En effet, supposons que $\bar{\zeta}_{\star_{\phi}}$ soit déterminé sur tous les mots de la forme $y_{1}^{i} y_{\mathbf{u}}$ avec $i \leq n$ et $y_{\mathbf{u}} \in Y_{c}$. On obtient, en supposant que $\bar{\zeta}_{\star_{\phi}}$ conserve la symétrie $\star_{\phi}$,

$$
\bar{\zeta}_{\star_{\phi}}\left(y_{1}\right) \bar{\zeta}_{\star_{\phi}}\left(y_{1}^{n} \mathbf{s}\right)=\bar{\zeta}_{\star_{\phi}}\left(y_{1}^{n+1} y_{\mathbf{s}}\right)+\ldots
$$

où ... est une somme de termes sur lesquels $\bar{\zeta}_{\star_{\phi}}$ est connue. On a donc

$$
\bar{\zeta}_{\star_{\phi}}\left(y_{1}^{n+1} y_{\mathbf{s}}\right)=\bar{\zeta}_{\star_{\phi}}\left(y_{1}\right) \bar{\zeta}_{\star_{\phi}}\left(y_{1}^{n} \mathbf{s}\right)+\ldots
$$

On définit donc $\bar{\zeta}_{\star_{\phi}}$ de manière unique, une constante $\gamma \in \mathbb{R}$ étant fixée pour $\bar{\zeta}_{\star_{\phi}}\left(y_{1}\right)$.

Pour être complet, il nous reste à comparer la régularisation directe que nous venons d'obtenir avec celle que l'on peut définir via la régularisation $\zeta_{\star}$ de $\zeta$.

Théorème 1. - Soit $\gamma \in \mathbb{R}$, on note $\zeta_{\star}$ et $\bar{\zeta}_{\star_{\phi}}$ les régularisés de $\zeta$ et $\bar{\zeta}$ respectivement tels que $\zeta_{\star}\left(y_{1}\right)=\bar{\zeta}_{\star_{\phi}}\left(y_{1}\right)=\gamma$. On a la relation

$$
\bar{\zeta}_{\star_{\phi}}\left(y_{\mathbf{s}}\right)=\zeta_{\star}\left(\phi\left(y_{\mathbf{s}}\right)\right) .
$$

Autrement dit, on peut effectuer la régularisation soit sur les $\bar{\zeta}$, soit sur leurs décompositions suivant les $\zeta$.

Démonstration. - Il suffit de démontrer que l'application $\zeta_{\star} \circ \phi$ est un élément qui vérifie les hypothèses du lemme 6 . Comme l'extension de $\bar{\zeta}$ est unique, on en déduira facilement l'identité (17).

On note $P\left(y_{\mathbf{u}}\right)=\zeta_{\star}\left(\phi\left(y_{\mathbf{u}}\right)\right)$ pour tout $y_{\mathbf{u}} \in Y^{*}$. On a $P\left(y_{\mathbf{s}}\right)=\bar{\zeta}\left(y_{\mathbf{s}}\right)$ pour tout $\mathbf{s} \in Y_{c}$ et $P\left(y_{1}\right)=\zeta_{\star}\left(\phi\left(y_{1}\right)\right)=\zeta_{\star}\left(y_{1}\right)=\gamma$ par hypothèse. Par ailleurs, pour tout $y_{\mathbf{s}}, y_{\mathbf{u}} \in Y^{*}$,

$$
P\left(y_{\mathbf{s}}\right) P\left(y_{\mathbf{u}}\right)=\zeta_{\star}\left(\phi\left(y_{\mathbf{s}}\right)\right) \zeta_{\star}\left(\phi\left(y_{\mathbf{u}}\right)\right)=\zeta_{\star}\left(\phi\left(y_{\mathbf{s}}\right) \star \phi\left(y_{\mathbf{u}}\right)\right)=P\left(y_{\mathbf{s}} \star_{\phi} y_{\mathbf{u}}\right) .
$$

Par conséquent, $P$ réalise bien une extension de $\bar{\zeta}$ sur les divergents respectant la symétrie $\star_{\phi}$. Par unicité, on en déduit $P=\bar{\zeta}_{\star_{\phi}}$.

\section{Formules de passage entre $\zeta$ et $\bar{\zeta}$}

On considère les séries génératrices de $\zeta$ et $\bar{\zeta}$ définies par

$$
\Phi_{\zeta}=\sum_{y_{\mathbf{s}} \in Y^{*}} \zeta_{\mathbf{s}} y_{\mathbf{s}} \text { et } \Phi_{\bar{\zeta}}=\sum_{y_{\mathbf{s}} \in Y^{*}} \bar{\zeta}_{\mathbf{s}} y_{\mathbf{s}},
$$

où $\zeta_{\mathbf{s}}\left(\operatorname{resp} . \bar{\zeta}_{\mathbf{s}}\right)$ est la valeur régularisée définie au paragraphe précédent.

Le lemme 4 et son extension via le théorème 1 se traduit par la relation suivante sur les séries génératrices :

Théorème 2. - On a $\Phi_{\bar{\zeta}}=\Phi_{\zeta} \circ 1$.

En effet, le coefficient de $\Phi_{\zeta} \circ \mathbf{1}$ est donné par

$$
\left(\Phi_{\zeta} \circ \mathbf{1}\right)_{\mathbf{s}}=\sigma \sum_{\mathbf{s}^{1} \ldots \mathbf{s}^{k}=\mathbf{s}} \zeta_{\left|\mathbf{s}^{1}\right|, \ldots,\left|\mathbf{s}^{k}\right|} \mathbf{1}_{\mathbf{s}^{1}} \ldots \mathbf{1}_{\mathbf{s}^{k}} .
$$

Comme $\mathbf{1}_{\mathbf{s}}=1$ pour toute suite $\mathbf{s}$, on en déduit le résultat. 
On peut inverser cette formule. En effet, on vérifie via le lemme 3 que la série $\mathbf{1}$ est inversible pour la loi o. Précisément, on a :

Lemme 7. - La série 1 a pour inverse de composition la série

$$
\mathbf{1}^{\circ-1}=\sum_{y_{\mathbf{s}} \in Y^{*}}(-1)^{l\left(y_{\mathbf{s}}\right)+1} y_{\mathbf{s}}
$$

où $l\left(y_{\mathbf{s}}\right)$ désigne la longueur de $y_{\mathbf{s}}$.

Démonstration. - Elle se fait par récurrence sur la longueur des suites. Pour une suite de longueur 1 , on a $\mathbf{1}_{s}^{\circ-1} \mathbf{1}_{s}=1$, soit $\mathbf{1}_{s}^{\circ-1}=1$ pour tout $s \in \mathbb{N}$. Par ailleurs, la relation $\mathbf{1}^{\circ-1} \mathbf{1}=\mathbf{I}$ donne pour toute suite $\mathbf{s}$ de longueur $l(\mathbf{s}) \geq 2$ :

$$
\mathbf{1}_{\mathbf{s}}^{\circ-1}+\sum_{\mathbf{s}^{1} \ldots \mathbf{s}^{k}=\mathbf{s}, 1 \leq k<l(\mathbf{s})} \mathbf{1}_{\left|\mathbf{s}^{1}\right|, \ldots,\left|\mathbf{s}^{k}\right|}^{\circ-1}=0 .
$$

Soit $p \geq 2$. Supposons $\mathbf{1}_{\mathbf{s}}^{\circ-1}=(-1)^{l(\mathbf{s})+1}$ pour toute suite de longueur $l(\mathbf{s}) \leq p$. On a donc pour une suite $\mathbf{s}$ de longueur $p+1$

$$
\mathbf{1}_{\mathbf{s}}^{\circ-1}=-\sum_{k=1}^{p}\left(\begin{array}{c}
p+1-k \\
p
\end{array}\right)(-1)^{k+1} .
$$

(Le coefficient $\left(\begin{array}{c}p+1-k \\ p\end{array}\right)$ représente le nombre de décomposition de la suite $\mathbf{s}$ en $k$ suites $\mathbf{s}^{1}, \ldots, \mathbf{s}^{k}$.) On en déduit le lemme.

On retrouve donc le résultat d'Ulanskii ([16, p. 578, Proposition 3]) sous une forme plus compacte.

Théorème 3. - Les séries génératrices $\Phi_{\bar{\zeta}}$ et $\Phi_{\zeta}$ vérifient la relation

$$
\Phi_{\zeta}=\Phi_{\bar{\zeta}} \circ 1^{\circ-1} \text {. }
$$

Cette formulation de la relation entre polyzêtas larges et stricts suggère d'étudier d'autres type de modification des polyzêtas stricts construites sur le même modèle, c'est à dire par composition via une série élémentaire.

\section{Les polyzêtas pondérés}

Le passage des inégalités strictes aux larges induit une modification des symétries. On peut se demander si il est possible de modifier de façon simple les polyzêtas strictes afin d'obtenir une symétrie de complexité réduite. Par exemple, est-il possible de modifier $\zeta$ tel que le nouvel objet vérifie la symétrie de battage?

Dans ce paragraphe, nous définissons les polyzêtas pondérés, d'expressions simples et de symétrie la symétrie de battage ou shuffle.

La symétrie vérifiée par $\zeta_{\text {s }}$ possède une traduction simple en terme algébrique. Elle traduit le caractère diagonal (on dit aussi group-like) de la série génératrice $\Phi_{\zeta}$ dans la cogèbre $\left(\mathbb{R}\langle Y\rangle, \Delta_{\star}\right)$, où $\Delta_{\star}$ est le coproduit défini par

$$
\Delta_{\star}\left(y_{s}\right)=\sum_{i+j=s} y_{i} \otimes y_{j}
$$

c'est-à-dire la relation

$$
\Delta_{\star}\left(\Phi_{\zeta}\right)=\Phi_{\zeta} \otimes \Phi_{\zeta} .
$$

On note $\Delta$ le morphisme $\mathrm{K}\langle Y\rangle \rightarrow \mathrm{K}\langle Y\rangle \otimes \mathrm{K}\langle Y\rangle$ défini par

$$
\Delta\left(y_{s}\right)=y_{s} \otimes 1+1 \otimes y_{s}
$$

pour tout $s \in \mathbb{N}$. 
On note Exp la série dite exponentielle définie par

$$
\operatorname{Exp}=\sum_{y_{\mathbf{s}} \in Y^{*}} \frac{1}{l(\mathbf{s}) !} y_{\mathbf{s}} .
$$

Lemme 8. - Soit $\Phi_{\mathrm{M}} \in \mathrm{K}\langle Y\rangle$ une série telle que $\Delta_{*}\left(\Phi_{\mathrm{M}}\right)=\Phi_{\mathrm{M}} \otimes \Phi_{\mathrm{M}}$, alors la série $\Phi_{\mathrm{M}, p}=$ $\Phi_{\mathrm{M}} \circ \operatorname{Exp}$ vérifie $\Delta\left(\Phi_{\mathrm{M}, p}\right)=\Phi_{\mathrm{M}, p} \otimes \Phi_{\mathrm{M}, p}$.

On renvoie à $[6]$ pour la démonstration.

Autrement dit, la composition par la série exponentielle Exp d'une série diagonale pour le coproduit $\Delta_{\star}$ donne une série diagonale pour le coproduit $\Delta$. La lettre $p$ indique que cette composition pondére par la longueur des mots le regroupement trivial $\Phi_{M} \circ \mathbf{1}$. Précisément, nous avons la formule

$$
\Phi_{\mathrm{M}, p}=\sum_{y_{\mathbf{s}} \in Y^{*}}\left(\sum_{\mathbf{s}^{1} \ldots \mathbf{s}^{k}=\mathbf{s}, k \geq 1} \frac{1}{l\left(\mathbf{s}^{1}\right) ! \ldots l\left(\mathbf{s}^{2}\right) !} \mathrm{M}_{\left|\mathbf{s}^{1}\right|, \ldots,\left|\mathbf{s}^{k}\right|}\right) y_{\mathbf{s}} .
$$

Le coproduit $\Delta$ est bien connu puisqu'il correspond à celui des algèbres de Hopf [13]. Dans ce cas, on montre que les éléments diagonaux vérifient la symétrie shuffle, notée ш et définie par récurrence sur la longueur des mots de $Y^{*}$ via les relations $y_{\mathbf{s}} \varpi \emptyset=y_{\mathbf{s}}, \emptyset ш y_{\mathbf{s}}=y_{\mathbf{s}}$ et

$$
y_{a} y_{\mathbf{s}} \amalg y_{b} y_{\mathbf{u}}=y_{a}\left(y_{\mathbf{s}} ш y_{b} y_{\mathbf{u}}\right)+y_{b}\left(y_{a} y_{\mathbf{s}} \amalg y_{\mathbf{u}}\right) .
$$

On remarque que cette symétrie est bien moins complexe que la symétrie stuffle (11) satisfaite par les polyzêtas stricts.

Nous sommes donc naturellement conduit à la définition des polyzêtas pondérés.

Définition 2. - Pour toute suite d'entier $\mathbf{s}$, on appelle polyzêta pondéré en $\mathbf{s}$ la quantité notée $\dot{\zeta}_{\mathbf{s}}$ et définie par

$$
\dot{\zeta}_{\mathbf{s}}=\sum_{\mathbf{s}^{1} \ldots \mathbf{s}^{k}=\mathbf{s}, k \geq 1} \frac{1}{l\left(\mathbf{s}^{1}\right) ! \ldots l\left(\mathbf{s}^{k}\right) !} \zeta_{\left|\mathbf{s}^{1}\right|, \ldots,\left|\mathbf{s}^{k}\right|}
$$

Le lemme 8 donne immédiatement que, pour tout mot $y_{\mathbf{s}}, y_{\mathbf{u}} \in Y^{*}$, on a

$$
\dot{\zeta}\left(y_{\mathbf{s}}\right) \dot{\zeta}\left(y_{\mathbf{u}}\right)=\dot{\zeta}\left(y_{\mathbf{s}} \amalg y_{\mathbf{u}}\right)
$$

Les polyzêtas pondérés sont utilisés par Jean Ecalle ([9, p. 418]) dans l'étude du prolongement méromorphe des multizêtas.

\section{Bibliographie}

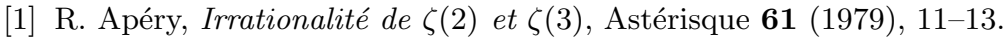

[2] W. N. Bailey, Generalized hypergeometric series, Cambridge University Press, Cambridge, 1935.

[3] K. Ball et T. Rivoal, Irrationalité d'une infinité de valeurs de la fonction zêta aux entiers impairs, Invent. Math. 146.1 (2001), 193-207.

[4] P. Cartier, Fonctions polylogarithmes, nombres polyzêtas et groupes pro-unipotents, Séminaire Bourbaki, Vol. 2000/2001, Astérisque 282 (2002), exposé No. 885, 137-173.

[5] P. Colmez, Arithmétique de la fonction zêta, Éd. École Polytechnique, 2003.

[6] J. Cresson, Calcul Moulien, Prépublication de l'I.H.E.S. 06/22 (2006), 93 pages.

[7] J. Cresson, S. Fischler et T. Rivoal, Séries hypergéométriques multiples et polyzêtas, Prépublication de l'I.H.E.S. 06/36 (2006).

[8] J. Cresson, S. Fischler et T. Rivoal, Phénomènes de symétrie dans des formes linéaires en polyzêtas, Prépublication de l'I.H.E.S. 06/37 (2006).

[9] J. Écalle, ARI/GARI, la dimorphie et l'arithmétique des multizêtas : un premier bilan, J. Théor. Nombres Bordeaux15 (2003), 411-478. 
[10] S. Fischler, Groupes de Rhin-Viola et intégrales multiples, J. Théor. Nombres Bordeaux 15 (2003), $\mathrm{n}^{\mathrm{o}} 2,479-534$.

[11] S. Fischler, Irrationalité de valeurs de zêta (d'après Apéry, Rivoal, ...), Séminaire Bourbaki, Vol. 2002/2003, Astérisque 294 (2004), exposé No. 910, 27-62.

[12] G. Racinet, Doubles mélanges des polylogarithmes multiples aux racines de l'unité, Publ. Math. Inst. Hautes Études Sci., 95 (2002), 185-231.

[13] C. Reutenauer, Free lie algebras, London Math. Soc. Monographs, new series 7, 1993.

[14] T. Rivoal, La fonction zêta de Riemann prend une infinité de valeurs irrationnelles aux entiers impairs, C. R. Acad. Sci. Paris, Série I Math. 331.4 (2000), 267-270.

[15] L. J. Slater, Generalized hypergeometric functions, Cambridge University Press, Cambridge, 1966.

[16] E. A. Ulanskiǔ, Identities for generalized polylogarithms (en russe), Mat. Zametki 73.4 (2003), 613-624; traduction en anglais dans Math. Notes 73 (2003), no. 3-4, 571-581.

[17] M. Waldschmidt, Valeurs zêtas multiples. Une introduction, J. Théor. Nombres Bordeaux 12 (2000), 581-595.

[18] M. Waldschmidt, Twisted Hoffman algebras, Report 12/2003, Colloque « Elementare und analytische Zahlentheorie », Oberwolfach, 2003.

[19] W. Zudilin, Arithmetic of linear forms involving odd zeta values, J. Théor. Nombres Bordeaux 16 (2004), 251-291.

8 novembre 2006

JaCky Cresson, Laboratoire de Mathématiques appliquées de Pau, Bâtiment I.P.R.A, Université de Pau et des Pays de l'Adour, avenue de l'Université, BP 1155, 64013 Pau cedex, France • E-mail : jacky.cresson@univ-pau.fr 